\title{
Lutar, esconder ou correr: como Tamandua tetradactyla (Mammalia) em cativeiro responde a pistas indiretas de um predador?
}

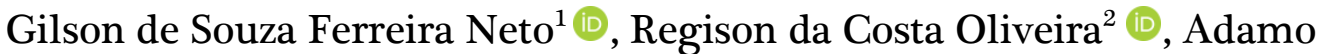 \\ Cardoso Barros $^{3}$ (D), Renato Pereira Ribeiro ${ }^{4}$, Raysa Melul ${ }^{5}$ (D) \& Fausto \\ Nomura $^{6,7,8}$ (D)
}

(1) Instituto Nacional de Pesquisas da Amazônia, Departamento de Ecologia, Programa de PósGraduação em Biologia (Ecologia), Avenida André Araújo 2936, Petrópolis 69067-375, Manaus, Amazonas, Brasil. E-mail: gilsonsouzaferreiraneto@gmail.com

(2) Instituto Nacional de Pesquisas da Amazônia, Programa de Pós-Graduação em Clima e Ambiente, Avenida André Araújo 2936, Petrópolis 69067-375, Manaus, Amazonas, Brasil.

(3) Tropical Sustainability Institute, Avenida Eliseu de Almeida 1578, Instituto da Previdência 05533000, São Paulo, Brasil. E-mail: adamobarros@gmail.com

(4) Instituto Federal de Educação, Ciência e Tecnologia do Maranhão, Avenida Getúlio Vargas, Monte Castelo 65030-005, São Luís, Maranhão, Brasil.

(5) Universidade Federal Rural da Amazônia, Laboratório de Pesquisa Morfológica Animal, Avenida Presidente Tancredo Neves 2501, Terra Firme 66077-830, Belém, Pará, Brasil.

(6) Universidade Federal de Goiás - Campus II, Instituto de Ciências Biológicas, Departamento de Ecologia, Avenida Esperança 1533, Campus Samambaia 74690-900, Goiânia, Goiás, Brasil.

(7) Universidade Federal de Goiás - Campus II, Instituto de Ciências Biológicas, Programa de PósGraduação em Ecologia e Evolução, Avenida Esperança, Campus Samambaia 74690-900, Goiânia, Goiás, Brasil.

(8) Universidade Federal de Goiás - Campus II, Instituto de Ciências Biológicas, Programa de PósGraduação em Biodiversidade Animal, Avenida Esperança, Campus Samambaia 74690-900, Goiânia, Goiás, Brasil.

Neto G.S.F., Oliveira R.G., Barros A.C., Ribeiro R.P., Melul R. \& Nomura F. (2021) Lutar, esconder ou correr: como Tamandua tetradactyla (Mammalia) em cativeiro responde a pistas indiretas de um predador? Pesquisa e Ensino em Ciências Exatas e da Natureza, 5: e1689.

http://dx.doi.org/10.29215/pecen.v5i0.1689

Editor acadêmico: Pablo Ariel Martinez. Recebido: 13 novembro 2020. Aceito: 30 março 2021. Publicado: 10 abril 2021

Resumo: No ambiente natural, os animais são expostos a uma grande quantidade de ameaças a sobrevivência. Considerar a ecologia do medo é importante para estimar o papel ecossistêmico que os predadores desempenham nas comunidades, uma vez que o medo de um predador pode provocar não somente respostas baseadas em densidade (i.e., remoção de indivíduos de uma população), como também respostas comportamentais, que podem resultar em efeitos em cascata. Comportamentos defensivos são relativamente simples de serem avaliados em situação de cativeiro, uma vez que são conspícuos e específicos, dificilmente sendo confundidos com outros comportamentos quando observados. Desta forma, o objetivo deste estudo foi verificar a habilidade de indivíduos de Tamandua tetradactyla (Linnaeus, 1758) em detectar pistas indiretas de um predador, para avaliação de risco do ambiente. Neste estudo, seis indivíduos de tamanduás-mirim ( $T$. tetradactyla) em cativeiro foram expostos a pistas indiretas (e.g., urina e fezes) de jaguatirica (Leopardus pardalis). No total, cinco machos e uma fêmea de tamanduá foram observados pelo método de animal focal, em um esforço total de 36 horas de observação. Tamanduás-mirins alocados nos recintos com pistas indiretas do predador exibiram maior frequência de comportamentos anti-predatórios e diminuição dos comportamentos de inatividade. A utilização de pistas indiretas é vantajosa para indivíduos de tamanduá-mirim, pois 
permitem a avaliação de risco de uma determinada mancha do ambiente e elicia comportamentos de evitação, que são menos custosos energeticamente. A porcentagem de comportamentos também é apresentada com e sem as pistas indiretas do predador de $T$. tetradactyla, na qual pode ser útil para entender a capacidade de resposta de tamanduás-mirins a situações de perigo.

Palavras chave: Ecologia do medo, tamanduá-mirim, jaguatirica, comportamentos anti-predatórios.

Fight, hide or run: how does Tamandua tetradactyla (Mammalia) in captivity respond to indirect predator clues?

Abstract: In the natural environment, animals are exposed to a wide number of threats to survival. Considering the ecology of fear is important to estimate the ecosystem role that predators play in animal communities, since fear of a predator can cause not only density-based responses (e.g., removal of individuals from a population), but also behavioural responses, which can result in cascading effects. Defensive behaviours are relatively simple to assess in captivity, since they are conspicuous and specific, and also difficult to be confused with other behaviours when observed. Thus, the aim of this study was to verify the ability of individuals of Tamandua tetradactyla (Linnaeus, 1758) in captivity to detect indirect clues of a natural predator. In this study, we exposed six individuals of lesser anteaters ( T. tetradactyla) in captivity, to indirect clues (e.g., urine and feces) of ocelot (Leopardus pardalis). In total, we observed five males and one lesser anteater female by the focal animal method, in a total effort of 36 hours of observation. Lesser anteaters located in enclosures with indirect predator tracks exhibited a higher frequency of anti-predatory behaviours and a decrease in inactivity behaviours. The use of indirect clues is advantageous for lesser anteater's individuals, as they allow the risk assessment of a particular spot in the environment and elicits avoidance behaviours, which are less costly in energy. We also present the percentage of behaviours of $T$. tetradactyla with and without the indirect clues of the predator that can be useful to understand the ability of lesser anteaters to respond to situations of danger.

Key words: Ecology of fear, lesser anteaters, ocelot, anti-predatory behaviours.

\section{Introdução}

Comportamentos defensivos são parte importante do repertório de qualquer organismo, sendo geralmente comportamentos complexos que exigem o reconhecimento de um contexto de risco e capacidade de resposta a situações de perigo (Navarro-Castilla \& Barja 2014). O efeito do medo pode provocar um custo em termos comportamentais, já que depende da capacidade da presa em avaliar o risco de permanecer em uma área para aproveitar oportunidades de alimentação ou fugir para reduzir as chances de ser atacado por um predador (Monclús et al. 2009). A capacidade de avaliar o risco afeta a atividade comportamental de indivíduos quando o predador não está presente no ambiente (Zanette \& Clinchy 2019). Tal como, após a extinção local dos lobos (Canis lupus) no Parque Nacional de Yellowstone na década de 1930, os alces (Cervus canadensis) passaram menos tempo vigilantes e começaram a se alimentar cada vez mais de gramíneas (Ripple \& Beschta 2012). Essa alteração de comportamento diminuiu a biomassa de plantas para outros mamíferos considerados como espécies-chave para o ecossistema, tal como o bisão americano (Bison bison) e o castor americano (Castor canadensis) (Ripple \& Beschta 2012). O efeito do medo que os predadores instigam nas presas é um fator ecológico importante que contribui para a manutenção dos ecossistemas naturais e pode causar alterações nas cadeias tróficas (Zanette \& Clinchy 2019). As manipulações experimentais em vertebrados terrestres são relativamente escassas, sendo os estudos focados em peixes (Ylönen et al. 2007; El Balaa \& Blouin-Demers 2011; Ullah et al. 2017), anfíbios (Nomura et al. 2011) e aves (Amo et al. 2011).

Apesar dos mamíferos serem um dos grupos mais bem estudados em termos de comportamentos, existem poucos estudos sobre o carismático Tamandua tetradactyla (Linnaeus 1758) (Catapani et al. 2019; Eguizábal et al. 2019; Neto et al. 2020). O tamanduá-mirim pode ser encontrado em uma variedade de ecossistemas, desde fragmentos do Cerrado brasileiro (Zimbres et al. 2013) a ilhas fluviais da Amazônia (Ferreira Neto et al. 2021). A espécie tem hábitos crepusculares (Rodrigues et al. 2001; Blake et al. 2012) e dieta especializada em cupins e 
formigas (Medri et al. 2006), contribuindo para a dinâmica do ecossistema (Montgomery 1985). Além disso, T. tetradactyla pode ser considerado uma espécie modelo para se estudar relações de predador-presa porque são presas de alguns dos predadores de topo mais importantes da região neotropical, como onças-pintadas (Panthera onca) (Foster et al. 2013), onças-pardas (Puma concolor) (Trovati \& Brito 2009) e jaguatiricas (L. pardalis) (Bianchi et al. 2014). Interações diretas entre predadores e presas são muito mais estudadas, porém os efeitos indiretos, como aqueles eliciados por pistas ambientais deixadas pela passagem do predador, são menos abordados, em particular, em mamíferos.

A capacidade de reconhecer o predador foi moldada por uma forte seleção evolutiva (Ferrero et al. 2011). Embora os predadores tenham evoluído para serem discretos e imprevisíveis enquanto estão caçando, urina e fezes são indicativos da presença do predador ou da possibilidade de ataque iminente e, por isso, estes indicativos são considerados medidas confiáveis para as presas avaliarem respostas anti-predatórias e reconhecerem ameaças (Hegab et al. 2015; Wernecke et al. 2015; Yin et al. 2017; St-Cyr et al. 2018), sendo tão eficientes quanto a própria presença do predador (Blanchard \& Blanchard 1989). Desta forma, o objetivo deste estudo foi avaliar o comportamento de indivíduos de $T$. tetradactyla em situação de cativeiro expostos a pistas indiretas de um predador natural. Assim, espera-se identificar mecanismos de avaliação de contexto de $T$. tetradactyla, e alterações no repertório comportamental, através de um aumento de comportamentos anti-predatórios.

\section{Material e Métodos}

\section{Área de estudo}

O estudo foi conduzido na fazenda Vale do Tamanduá (16 59'25.2" S, 49²4'17.2" O), município de Aragoiânia, Goiás, Brasil (Figura 1). A fazenda é um centro de vida selvagem que reabilita animais em cativeiro. $\mathrm{O}$ tamanho da fazenda é de 99 hectares e a área da fazenda inclui vegetação nativa e diferentes fisionomias de Cerrado (Eiten 1978).

\section{Indivíduos amostrados}

Todos os indivíduos observados de T. tetradactyla foram resgatados do bioma Cerrado de diferentes áreas do estado de Goiás por veterinários do Centro de Triagem de Animais Silvestres (CETAS) de Goiânia, gerido pelo Instituto Brasileiro do Meio Ambiente e dos Recursos Naturais Renováveis (IBAMA), antes de serem transferidos para recintos na fazenda Vale do Tamanduá. Um total de seis indivíduos foram analisados no estudo, sendo um infante (2-3 meses), dois machos jovens (9-15 meses), dois machos adultos (24-60 meses) e uma fêmea jovem (12 meses). Um macho jovem de jaguatirica (Leopardus pardalis) foi utilizada como modelo de predador. Tal indivíduo foi resgatada pelo CETAS-GO e também transferido para a fazenda Vale do Tamanduá para ser reabilitado por veterinários.

$\mathrm{O}$ indivíduo de jaguatirica foi usado neste experimento por atuar como um dos principais predadores naturais de tamanduás (Bianchi et al. 2014). Jaguatiricas e tamanduás possuem hábitos noturnos em vida livre (Rodrigues et al. 2001; Blake et al. 2012) e ambas as espécies têm preferência por habitats florestais em alguns biomas (Pardo Vargas 2018). As jaguatiricas são mesopredadores de médio porte e carnívoros especialistas $(>10 \mathrm{~kg})$ que se alimentam de diversos tipos de vertebrados, principalmente de pequenos mamíferos, cobras e roedores. Tamanduás mirins $(>1.5 \mathrm{~kg}$ ) também são parte importante da dieta de jaguatiricas (Bianchi et al. 2014).

Os tamanduás foram facilmente reconhecidos individualmente devido à variação na coloração e tamanho do corpo. Em cada sessão de observações, dois indivíduos de tamanduámirim, escolhidos aleatoriamente, foram inseridos em um recinto de $2.72 \mathrm{~m}$ (comprimento) $\mathrm{x}$ $2.40 \mathrm{~m}$ (largura) x $3.94 \mathrm{~m}$ (altura). Os animais foram observados em pares para permitir a emissão de comportamentos sociais. $\mathrm{O}$ alimento foi oferecido gradativamente para amenizar 
possíveis efeitos negativos da restrição de espaço (Catapani et al. 2019). O alimento e a água foram constantemente renovados todas as manhãs.

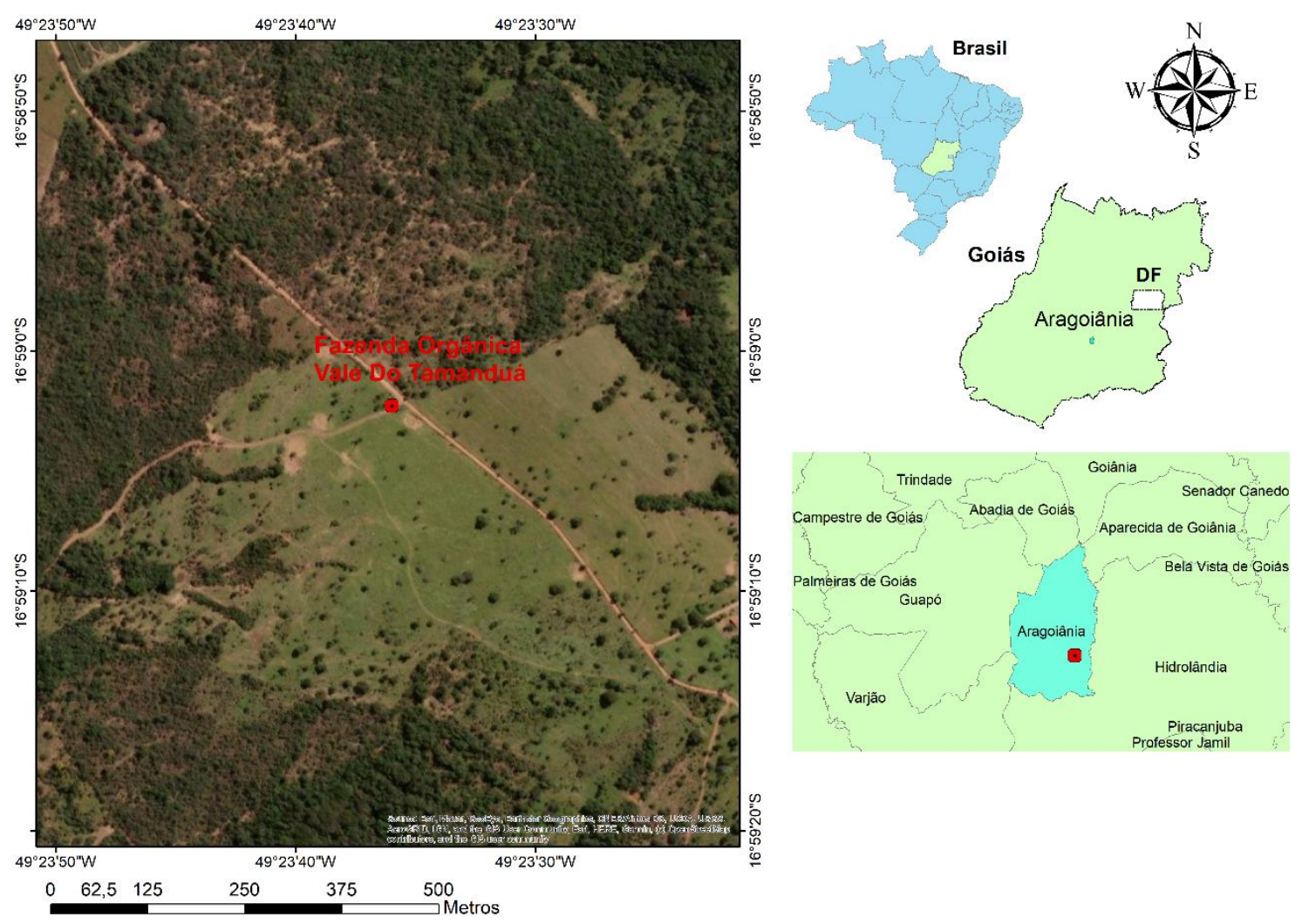

Figura 1. Mapa da área de estudo no município de Aragoiânia, Goiás, Brasil.

\section{Coleta de comportamentos}

Observações experimentais foram feitas durante três finais de semana, totalizando 36 horas de amostragem, sendo 18 horas de amostragem em um recinto em que o predador não havia sido previamente inserido (recinto sem os odores do predador) e 18 horas em um recinto na qual a jaguatirica havia sido previamente inserida (recinto com os odores do predador) e retirada somente após defecar e urinar. Todos os seis indivíduos de tamanduás (três pares) foram submetidos a ambos os tratamentos, sendo seis horas em cada tratamento. Desta forma, a pesquisa avaliou se o repertório comportamental dos tamanduás foi modificado em função da percepção do risco de predação com base em pistas indiretas (e.g., urina, fezes, odor da pelagem, secreções da glândula anal) da presença de Leopardus pardalis. Observações preliminares foram feitas dois meses antes da coleta de dados, para minimizar o viés do observador e para os observadores se familiarizarem com a identidade dos indivíduos. Antes do monitoramento, randomizou-se quais indivíduos seriam observados com e sem as pistas indiretas do predador. A amostragem animal focal foi usada, na qual as ocorrências de comportamentos são registradas continuamente (Altmann 1974). O monitoramento ocorreu entre 06:00 às 12:00, no período de maior atividade desses indivíduos em cativeiro (Neto et al. 2020). Quatro observadores trabalharam em pares e alternaram entre os fins de semana. A jaguatirica e os tamanduás foram transferidos de um recinto a outro contidos em espaço chamado de cambeamento. $O$ estudo obedeceu à legislação brasileira e a autorização para o estudo foi recebida do CETAS-GO.

\section{Análise de dados}

Neste estudo, a frequência dos comportamentos de T. tetradactyla $(\mathrm{n}=6)$ foi modelada em função da variável categórica ou efeitos fixos: as pistas indiretas da jaguatirica. Para isso, foi usado um modelo misto (Zuur et al. 2009), visto que os dados não foram independentes (Winter 2013). Posteriormente, aplicou-se a função ANOVA nos modelos aninhados como um teste de 
hipótese para obter o valor de p e testar se cada um dos modelos com os comportamentos foi diferente do modelo nulo. Desta forma, a pesquisa verificou se as pistas indiretas de jaguatirica influenciaram os comportamentos de tamanduás. A soma de todos os comportamentos individuais por categoria foi usada para todos os indivíduos e os comportamentos foram classificados em: alimentação, defesa, forrageamento, inatividade, limpeza e locomoção adaptado de Neto et al. (2020). O estudo empregou o pacote lme4 (Bates et al. 2014) no R versão 3.3.1 (R Core Team 2020).

\section{Resultados}

Um total de seis categorias comportamentais foram observados (Figura 2, Tabela 1): i) alimentação - que inclui a dieta oferecida por veterinários, alimento encontrado em ambiente natural como cupins e formigas e também beber água; ii) defesa - que inclui atividades como levantar, na qual os indivíduos adotam uma posição característica dos tamanduás, formada pela cauda e membros posteriores voltados para o possível adversário. Nesta categoria, foram observados também comportamentos de esconder no solo, em troncos ou cupinzeiros, além de se locomover mais rapidamente tentando escapar em substrato arbóreo e terrestre; iii) forrageamento - que inclui atividades de inspeção do ambiente, atividades de procura por alimento em substrato arbóreo e no solo; iv) inatividade - que inclui atividades de descanso; v) limpeza - que inclui atividades como coçar, defecar e urinar; e vi) locomoção - que inclui atividade de deslocamento.

Um aumento na frequência de comportamentos de defesa foi observado quando pistas indiretas do predador estavam presentes no recinto, aumentando de $2.72 \%$ para $22.44 \%$ do total de comportamentos exibidos (Tabela 1). Uma emissão do comportamento de atividade lúdica foi observada somente no recinto onde o predador não foi inserido, por isso, este comportamento não foi contabilizado nas análises, no qual o indivíduo coloca a língua para fora depois de ter se alimentado (Figura 2).

Os comportamentos observados nesse estudo foram moldados pelo reconhecimento do odor do predador. Um aumento significativo da frequência de comportamentos de defesa e limpeza e uma diminuição dos comportamentos de inatividade foram observados. Para os outros comportamentos, como alimentação, forrageamento e locomoção, não houve diferenças significativas entre os dois tratamentos (Tabela 2).

Tabela 1. Porcentagem de comportamentos observados para seis indivíduos de Tamandua tetradactyla em cativeiro na fazenda Vale do Tamanduá, Aragoiânia, Goiás, Brasil.

\begin{tabular}{|c|c|c|c|}
\hline Categoria comportamental & Comportamento & $\begin{array}{c}\text { Porcentagem sem as } \\
\text { pistas }\end{array}$ & $\begin{array}{c}\text { Porcentagem com as } \\
\text { pistas }\end{array}$ \\
\hline \multirow{4}{*}{ Alimentação } & Come cupim de monte & $12.92 \%$ & $7.48 \%$ \\
\hline & Bebe água & $0.68 \%$ & $0 \%$ \\
\hline & Come dieta oferecida no recinto & $4.76 \%$ & $4.76 \%$ \\
\hline & Come formigas & $0 \%$ & $2.72 \%$ \\
\hline \multirow{4}{*}{ Defesa } & Levantar & $2.72 \%$ & $7.48 \%$ \\
\hline & Esconder no tronco e no solo & $0 \%$ & $4.08 \%$ \\
\hline & Correr & $0 \%$ & $8.84 \%$ \\
\hline & Se mover pela grade tentando sair & $0 \%$ & $2.04 \%$ \\
\hline \multirow{3}{*}{ Forrageamento } & Forrageia cupim de árvore & $8.16 \%$ & $2.72 \%$ \\
\hline & Forrageia cupim de solo & $6.80 \%$ & $20.40 \%$ \\
\hline & Farejando o ambiente & $10.20 \%$ & $25.85 \%$ \\
\hline Inatividade & Descansa & $22.44 \%$ & $0 \%$ \\
\hline \multirow{3}{*}{ Limpeza } & Se coça & $12.24 \%$ & $0.68 \%$ \\
\hline & Urinar & $0.00 \%$ & $4.76 \%$ \\
\hline & Defecar & $0.00 \%$ & $1.36 \%$ \\
\hline \multirow{2}{*}{ Locomoção } & Desloca no solo & $17 \%$ & $4.08 \%$ \\
\hline & Desloca na árvore & $2.04 \%$ & $2.72 \%$ \\
\hline
\end{tabular}




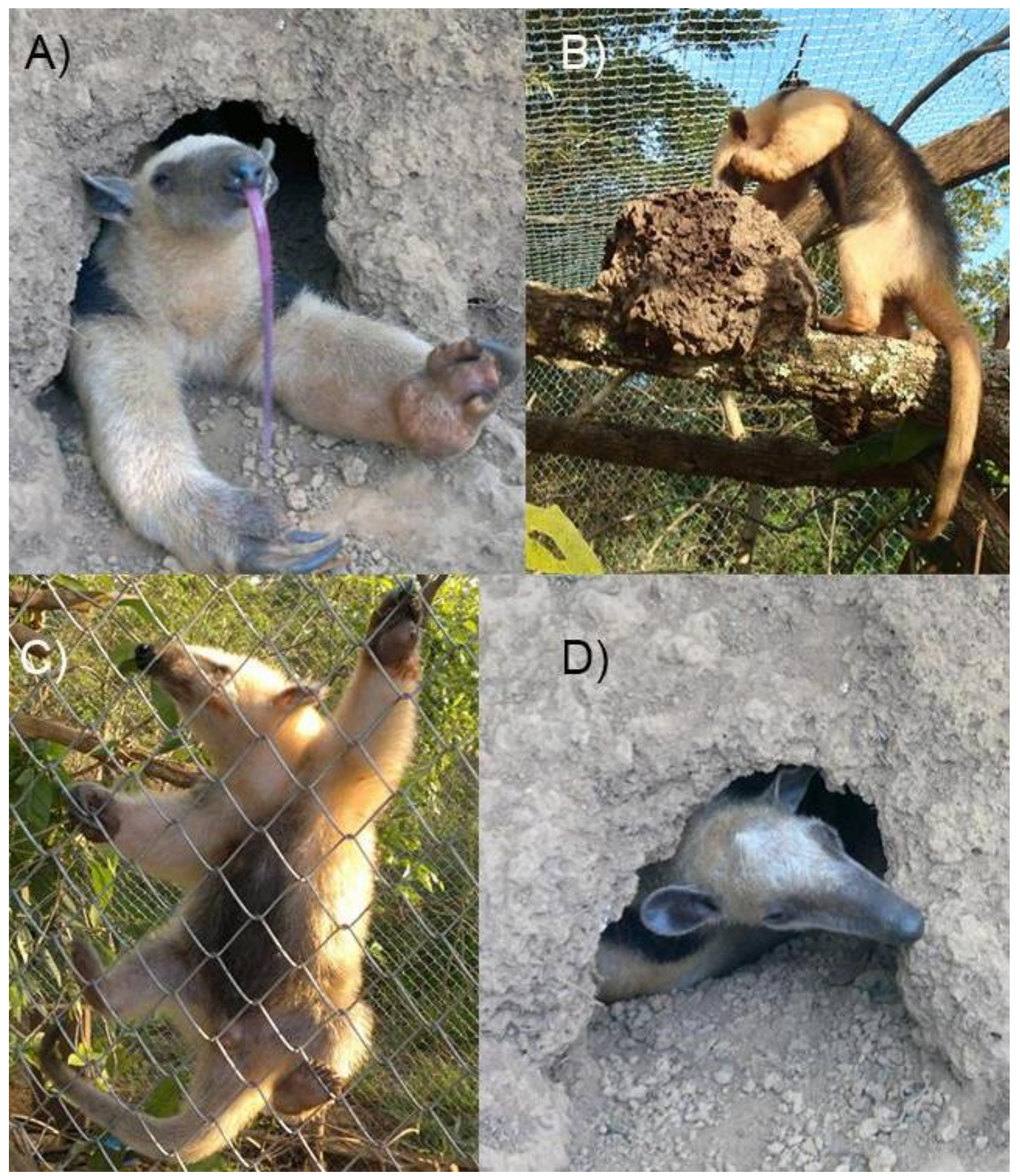

Figura 2. Exemplos de comportamentos observados para Tamandua tetradactyla em cativeiro: A. Atividade lúdica após o indivíduo ter se alimentado de cupins; B. Alimentando de cupim de árvore; C. Indivíduo sobe na grade tentando escapar ao reconhecer o odor do predador; D. Indivíduo se esconde na toca e observa atentamente a possibilidade da presença de um potencial predador. Comportamentos A e B ocorreram no recinto sem as pistas indiretas do predador, enquanto que os comportamentos $\mathrm{C}$ e D ocorreram no recinto com as pistas indiretas do predador.

Tabela 2. Resumo dos resultados dos modelos mistos mostrando a influência de pistas indiretas de um predador natural no comportamento dos tamanduás-mirins em cativeiro $(\mathrm{n}=6)$. DP = desvio padrão, $\mathrm{C}$. angular $=$ Coeficiente angular. Em negrito, os comportamentos significativos $(\mathrm{p}<0.05)$.

\begin{tabular}{lccccc}
\hline \multirow{2}{*}{ Comportamentos } & \multicolumn{2}{c}{ Efeitos fixos } & \multicolumn{2}{c}{ Efeitos aleatórios } & Teste de anova \\
\cline { 2 - 6 } & Intercepto & C. angular & DP & Variância & Pr (> Chisq) \\
\hline Alimentação & 4.83 & -0.33 & 0.70 & 0.50 & 0.65 \\
\hline Defesa & 5.50 & -5.33 & 0.83 & 0.70 & 0.0006 \\
\hline Forrageamento & 6.83 & -0.66 & 0 & 0 & 0.80 \\
\hline Inatividade & $-1.54 \mathrm{E}-12$ & 5500 & 0.10 & 0.011 & 0.01 \\
\hline Limpeza & 1.66 & 3 & 0.40 & 0.63 & 0.01 \\
\hline Locomoção & 1.66 & 1.33 & 1.21 & 1.46 & 0.06 \\
\hline
\end{tabular}




\section{Discussão}

As pistas indiretas de um possível predador suprimiram comportamentos de inatividade como dormir e descansar geralmente observados em tamanduá-mirim em cativeiro (Neto et al. 2020), o que corrobora outros estudos com diferentes grupos biológicos envolvendo invertebrados a peixes e mamíferos, nos quais alguns comportamentos geralmente observados foram suprimidos com as pistas indiretas de predadores naturais (Sih et al. 1992; Chivers \& Smith 1994; Hill \& Lodge 1994; Korpimaki et al. 1996; Lima 1998). O mesmo resultado também foi verificado em ratazanas que nunca tiveram experiência com odor de predadores como raposas, mas aumentaram a quantidade de comportamentos anti-predatórios (Wernecke et al. 2015), assim como cervos (Cervus nippon), submetidos a compostos orgânicos presentes no odor de lobos japoneses (Canis lupus), cuja subespécie no Japão foi extinta há mais de 120 anos (Osada et al. 2014). Da mesma forma, demonstrou-se neste estudo um aumento na frequência de comportamentos anti-predatórios de indivíduos de $T$. tetradactyla, como locomover rapidamente na tentativa de fugir, esconder-se em troncos e buracos e levantar-se. Esses comportamentos de defesa são característicos dos tamanduás quando se sentem ameaçados por predadores (Medri et al. 2006). Uma das estratégias anti-predatórias mais comuns observadas foi o comportamento de se levantar, na qual indivíduos de $T$. tetradactyla assumem uma postura em tripé, formada pelas patas traseiras e cauda, com membros anteriores estendidos e garras livres para defesa (Medri et al. 2006). As unhas dos membros anteriores e posteriores, além de terem um papel importante na abertura de formigueiros e cupinzeiros, podem também servir como defesa (Nowak \& Paradiso 1983). No presente estudo, também observou-se tamanduás se escondendo em buracos e troncos. A estratégia dos tamanduás de se esconder os tornam menos suscetíveis as constantes queimadas do bioma Cerrado, e é uma forma de proteção contra predadores naturais (Montgomery \& Lubin 1977; Redford 1984; Redford 1994). De modo geral, os indivíduos ficaram mais tempo vigilantes, e por isso descansaram menos. A frequência dos comportamentos de defesa de $T$. tetradactyla aumentaram na presença de pistas indiretas do predador, o que representa a capacidade de avaliação de risco. Além disso, houve um aumento significativo de comportamentos de limpeza como defecar e urinar, quando os animais foram colocados na presença de pistas indiretas. Consequentemente, esses resultados indicam que os tamanduás identificaram e reagiram as pistas indiretas, através do reconhecimento do odor natural do predador.

A obtenção de dados comportamentais básicos é crítica para a conservação das espécies. Os comportamentos observados aqui foram avaliados por um período de tempo curto. Outros trabalhos demonstraram que a exposição por um período mais curto à pistas indiretas de predadores promoveram aumento de comportamentos defensivos em ratazanas (Rattus norvegicus), mas durante uma exposição mais prolongada as pistas indiretas do predador, os comportamentos anti-predatórios diminuíram ou acabaram (Yin et al. 2017). Uma possível razão para isso é porque as atividades de vigilância, apesar de serem essenciais para qualquer organismo, demandam muita energia e, caso a presa reconheça que as pistas indiretas não irão ameaçá-la, pode ser mais vantajoso recuperar a homeostase e economizar essa energia para outras demandas como alimentação e forrageamento (Monclús et al. 2009). Alguns poderiam afirmar que tais comportamentos defensivos seriam uma resposta a qualquer odor diferente. No entanto, pequenos roedores não aumentaram a quantidade de comportamentos anti-predatórios ao serem submetidos ao odor de herbívoros, mas mostraram esses comportamentos de defesa para os odores de felinos e canídeos (Fendt 2006), o que reforça a possibilidade de tais comportamentos serem inatos, mesmo para animais em cativeiro. No entanto, em um ambiente natural, as pistas indiretas são reconhecidas apenas por presas nativas que coevoluíram com o predador natural, mas não por presas cujos predadores foram introduzidos recentemente. Este é o caso de vários roedores na Austrália que ainda não conseguem identificar as pistas indiretas de invasores exóticos como a raposa (Vulpes vulpes), como um sinal de ameaça, ao contrário das presas européias, onde a raposa é um predador natural (Dickman \& Doncaster 1984; Banks 1998). Logo, o reconhecimento imediato do predador com um comportamento inato ou 
aprendido, é uma vantagem para a sobrevivência dos indivíduos, visto que a presa poderia facilmente ser predada no ambiente natural com comportamentos anti-predatórios inadequados (Fendt 2006). Caso a presa não conseguisse perceber o odor do predador em cativeiro, seria necessário treinar os indivíduos para reconhecerem e serem mais vigilantes no ambiente que for reintroduzida, e desta forma, aumentar as chances de sobrevivência, especialmente de espécies emblemáticas e ameaçadas de extinção (McLean 1997; Mathews et al. 2005).

A partir destes resultados, o trabalho levanta informações em relação as melhores práticas de reabilitação em cativeiro para o tamanduá-mirim, antes da soltura definitiva, e reforça a importância de comportamentos de vigilância adequados de animais em cativeiro para aumentar as chances de sucesso de reintroduções e translocações (Whitwell et al. 2012). Desta forma, sugere-se experimentos manipulativos que levem em consideração mais indivíduos para avaliar como o medo de um predador pode afetar a capacidade de aprendizado e resposta defensiva dos comportamentos da presa. Por exemplo, pode ser importante para tomada de decisão, proteger cupinzeiros se forem usados para marcação de território por predadores, eliciando interações indiretas positivas entre tamanduás e cupins ou formigas. Também seria interessante avaliar em estudos futuros se tamanduás podem discriminar entre diferentes tipos de predadores, por exemplo, se pistas indiretas de onças provocam uma resposta mais intensa em relação a jaguatiricas ou mesmo abordagens que utilizem estímulos visuais do predador. Outra possibilidade seria considerar mudanças comportamentais e neuroendócrinas da presa que estão intrinsicamente relacionadas, para um melhor entendimento das interações predadorpresa. No ambiente natural, sugere-se avaliar como a paisagem do medo pode suprimir os comportamentos da presa considerando a variação espacial e temporal, além da magnitude dos efeitos do medo nas comunidades biológicas.

\section{Agradecimentos}

Agradecemos a Beth e Telmo, proprietários da área onde os estudos foram realizados, pelo apoio logístico e ajuda neste trabalho. Agradecemos aos assistentes de campo Marco Prado, Pedro Ubatan e Sandro Júnior. Finalmente, agradecemos ao Centro de Triagem de Animais Silvestres de Goiás (CETAS/IBAMA-GO) pela autorização e apoio para a realização deste estudo.

\section{Referências}

Altmann J. (1974) Observational study of Behaviour: sampling methods. Behaviour, 49: 227-266. https://doi.org/10.1163/156853974X00534

Amo L., Visser M.E. \& Van Oers K. (2011) Smelling out predators is innate in birds. Ardea, 99: 177-184. https://doi.org/10.5253/078.099.0207

Banks P.B. (1998) Responses of Australian bush rats, Rattus fuscipes, to the odor of introduced Vulpes vulpes. Journal of Mammalogy, 79: 1260-1264. https://doi.org/10.2307/1383017

Bates D., Mächler M., Bolker B. \& Walker S. (2014) Fitting linear mixed-effects models using lme4. arXiv preprint arXiv: 1406.5823.

Bianchi R.D.C., Campos R.C., Xavier-Filho N.L., Olifiers N., Gompper M.E. \& Mourão G. (2014) Intraspecific, interspecific, and seasonal differences in the diet of three mid-sized carnivores in a large neotropical wetland. Acta Theriologica, 59: 13-23. https://doi.org/10.1007/s13364013-0137-x

Blake J.G., Mosquera D., Loiselle B.A., Swing K., Guerra J. \& Romo D. (2012) Temporal activity patterns of terrestrial mammals in lowland rainforest of eastern Ecuador. Ecotropica, 18: 137-146.

Blanchard R.J. \& Blanchard D.C. (1989) Antipredator defensive behaviors in a visible burrow system. Journal of Comparative Psychology, 103: 70-82. https://doi:10.1037/0735-7036.103.1.70

Caro S.P. \& Balthazart J. (2010) Pheromones in birds: myth or reality? Journal of Comparative Psychology A, 196: 751-766. https://doi.org/10.1007/s00359-010-0534-4 
Catapani M.L., Pires J.S.R. \& Vasconcellos A.D.S. (2019) Single-or Pair-Housed: Which Is Better for Captive Southern Tamanduas? Journal of Applied Animal Welfare Science, 22: 289-297. https://doi.org/10.1080/10888705.2018.1508352

Chivers D.P. \& Smith R.J.F. (1994) Fathead minnows, Pimephales promelas, acquire predator recognition whe alarm substance is associated with the sight of unfamiliar fish. Animal Behaviour, 48: 597-605. https://doi.org/10.1006/anbe.1994.1279

Dickman C.R. \& Doncaster C.P. (1984) Responses of small mammals to red fox (Vulpes vulpes) odour. Journal of Zoology, 204: 521-531. https://doi.org/10.1111/j.1469-7998.1984.tb02384.x

Eguizábal G.V., Palme R.M., Superina C.J., Asencio M.C., García C. \& Busso J.M. (2019) Characterization and correlations of Behavioural and adrenocortical activities of zoo-housed lesser anteaters (Tamandua tetradactyla). Zoo Biology, 38: 334-342. https://doi.org/10.1002/zoo.21492

Eiten G. (1978) Delimitation of the cerrado concept. Vegetatio, 36: 169-178. https://doi.org/10.1007/BF02342599

El Balaa R. \& Blouin-Demers G. (2011) Anti-predatory behaviour of wild-caught vs captive-bred freshwater angelfish, Pterophyllum scalare. Journal of Applied Ichthyology, 27: 1052-1056. https://doi.org/10.1111/j.1439-0426.2011.01740.x

Fendt M. (2006) Exposure to urine of canids and felids, but not of herbivores, induces defensive behavior in laboratory rats. Journal of Chemical Ecology, 32: 2617-2627. https://doi: 10.1007/s10886-006-9186-9

Ferreira Neto G.D.S., Baccaro F.B., Spironello W.R., Benchimol M., Fleischer K., Quesada C.A., Sousa Gonçalves A.L., Pequeno P.A.L. \& Barnett A.P.A. (2021) Soil fertility and anthropogenic disturbances drive mammal species richness and assemblage composition on tropical fluvial islands. Austral Ecology. https://doi:10.1111/aec.13023

Ferrero D.M., Lemon J.K., Fluegge D., Pashkovski S.L., Korzan W.J., Datta S.R., Spehr M., Fendt M. \& Liberles S.D. (2011) Detection and avoidance of a carnivore odor by prey. Proceedings of the National Academy of Sciences, 108: 11235-11240. https://doi.org/10.1073/pnas.1103317108

Foster V.C., Sarmento P., Sollmann R., Tôrres N., Jácomo A.T., Negrões N. \& Silveira L. (2013) Jaguar and puma activity patterns and predator-prey interactions in four Brazilian biomes. Biotropica, 45: 373-379. https://doi.org/10.1111/btp.12021

Hegab I.M., Kong S., Yang S., Mohamaden W.I. \& Wei W. (2015) The ethological relevance of predator odors to induce changes in prey species. Acta Ethologica, 18: 1-9. https://doi.org/10.1007/s10211-014-0187-3

Hill A.M. \& Lodge D.M. (1994) Diel changes in resource demand: competition and predation in species replacement among crayfishes. Ecology, 75: 2118-2126. https://doi.org/10.2307/1941615

Korpimaki E., Koivunen V. \& Hakkarainen H. (1996) Microhabitat use and behavior of voles under weasel and raptor predation risk: predator facilitation? Behavioral Ecology, 7: 30-34. https://doi.org/10.1093/beheco/7.1.30

Lima S.L. (1998) Nonlethal effects in the ecology of predator-prey interactions. Bioscience, 48: 25-34. https://doi.org/10.2307/1313225

Mathews F., Orros M., McLaren G., Gelling M. \& Foster R. (2005) Keeping fit on the ark: assessing the suitability of captive-bred animals for release. Biological Conservation, 121: 569-577. https://doi.org/10.1016/j.biocon.2004.06.007

McLean I.G. (1997) Conservation and the ontogeny of behaviour (p. 132-156). In: Clemmons J.R. \& Buchholz R. (Eds). Behavioral Approaches to Conservation in the Wild. Cambridge: Cambridge University Press. 404 p.

Medri I.M., Mourão G.M. \& Rodrigues F.H. (2006) Mamíferos do Brasil. Londrina: Universidade Estadual de Londrina. 437 p.

Monclús R., Palomares F., Tablado Z., Martinez-Fonturbel A. \& Palme R. (2009) Testing the threat-sensitive predator avoidance hypothesis: physiological responses and predator pressure in wild rabbits. Oecologia, 158: 615-623. https://doi.org/10.1007/s00442-008-1201-0 
Montgomery G.G. (1985) Movements, foraging and food habits of the four extant species of neotropical vermilinguas (Mammalia: Myrmecophagidae) (p. 365-377). In: Montgomery G.G. (Ed.). The Evolution and Ecology of Armadillos, Sloths and Vermilinguas. Washington, DC.: Smithsonian Institution Press. $451 \mathrm{p}$.

Montgomery G.G. \& Lubin Y.D. (1977) Prey influences on movements of Neotropical anteaters. In: Proceedings of the 1975 predator symposium. Missoula: Montana Forest and Conservation Experiment Station, University of Montana.

Navarro-Castilla Á. \& Barja I. (2014) Does predation risk, through moon phase and predator cues, modulate food intake, antipredatory and physiological responses in wood mice (Apodemus sylvaticus)? Behavioral Ecology and Sociobiology, 68: 1505-1512. https://doi.org/10.1007/s00265-014-1759-y

Neto G.S.F., Barros A.C., Sobroza T.V., Neves P.U.C., Phillips M.J. \& Guimarães E.F. (2020) Period of the day and food-based enrichment affect behaviour activity of Tamandua tetradactyla in captivity? Pesquisa e Ensino em Ciências Exatas e da Natureza, 4: e1498. http://dx.doi.org/10.29215/pecen.v4i0.1498

Nomura F., Prado D., Silva V.H.M., Borges F.R., Dias R.E. \& Rossa-Feres D.D.C. (2011) Are you experienced? Predator type and predator experience trade-offs in relation to tadpole mortality rates. Journal of Zoology, 284: 144-150. https://doi.org/10.1111/j.1469-7998.2011.00791.x

Nowak RM. \& Paradiso J. L. (1983) Walker's mammals of the world. $4^{\circ}$ edition. Baltimore and London: The Johns Hopkings Press. 1470 p.

Osada K., Miyazono S. \& Kashiwayanagi M. (2014) Pyrazine analogs are active components of wolf urine that induce avoidance and fear-related behaviors in deer. Frontiers in Behavioral Neuroscience, 8: 276. https://doi.org/10.3389/fnbeh.2014.00276

Pardo Vargas L.E. (2018) Diversity and habitat use of medium-large sized mammals across oil palm landscapes in the Llanos region of Colombia. Thesis, College of Science and Engineering, James Cook University, Queensland, Australia. https://doi.org/10.25903/5be8adb35232e

R Core Team (2020) R: A Language and Environment for Statistical Computing. R Foundation for Statistical Computing, Vienna, Austria. https://www.R-project.org

Redford K.H. (1984) Mammalian myrmecophagy: feeding, foraging and food preference. Ph.D. dissertation, Harvard University, Cambridge, MA. 299 p.

Redford K.H. (1994) The edentates of the Cerrado. Edentata, 1: 4-10.

Ripple W.J. \& Beschta R.L. (2012) Trophic cascades in Yellowstone: the first 15 years after wolf reintroduction. Biological Conservation, 145: 205-213. https://doi.org/10.1016/j.biocon.2011.11.005

Rodrigues F.H., Marinho-Filho J. \& Dos Santos H.G. (2001) Home ranges of translocated lesser anteaters Tamandua tetradactyla in the cerrado of Brazil. Oryx, 35: 166-169. https://doi.org/10.1046/j.1365-3008.2001.00162.x

Sih A., Kats L.B. \& Moore R.D. (1992) Effects of predatory sunfish on the density, drift, and refuge use of stream salamander larvae. Ecology, 73: 1418-1430. https://doi.org/10.2307/1940687

St-Cyr S., Abuaish S., Spinieli R.L. \& McGowan P.O. (2018) Maternal predator odor exposure in mice programs adult offspring social behavior and increases stress-induced behaviors in semi-naturalistic and commonly-used laboratory tasks. Frontiers in Behavioral Neuroscience, 12: 136. https://doi.org/10.3389/fnbeh.2018.00136

Trovati R.G \& Brito B.D. (2009) Nota sobre deslocamento e área de uso de tamanduá-mirim (Tamandua tetradactyla) translocado no Cerrado brasileiro. Neotropical Biology and Conservation, 4: 144-149. http://dx.doi.org/10.4013/nbc.2009.43.04

Ullah I., Zuberi A., Khan K.U., Ahmad S., Thörnqvist P.O. \& Winberg S. (2017) Effects of enrichment on the development of behaviour in an endangered fish mahseer (Tor putitora). Applied Animal Behaviour Science, 186: 93-100. https://doi.org/186:93-100. 10.1016/j.applanim.2016.10.016 
Wernecke K.E.A., Vincenz D., Storsberg S., D’Hanis W., Goldschmidt J. \& Fendt M. (2015) Fox urine exposure induces avoidance behavior in rats and activates the amygdalar olfactory cortex. Behavioural Brain Research, 279: 76-81. https://doi.org/10.1016/j.bbr.2014.11.020

Whitwell S.M., Amiot C., Mclean I.G., Lovegrove T.G., Armstrong D.P., Brunton D.H. \& Ji W. (2012) Losing anti-predatory behaviour: A cost of translocation. Austral Ecology, 37: 413-418. https://doi.org/10.1111/j.1442-9993.2011.02293.x

Winter B. (2013) Linear models and linear mixed effects models in R: Tutorial 11. arXiv preprint arXiv:1308.5499.

Yin B., Gu C., Lu Y., Hegab I.M., Yang S., Wang A. \& Wei W. (2017) Repeated exposure to cat urine induces complex behavioral, hormonal, and c-fos mRNA responses in Norway rats (Rattus norvegicus). The Science of Nature, 104: 1-8. https://doi.org/10.1007/s00114-017-14842

Ylönen H., Kortet R., Myntti J. \& Vainikka A. (2007) Predator odor recognition and antipredatory response in fish: does the prey know the predator diel rhythm? Acta Oecologica, 31: 1-7. https://doi.org/10.1016/j.actao.2005.05.007

Zanette L.Y \& Clinchy M. (2019) Ecology of fear. Current Biology, 29: 309-313. https://doi.org/10.1016/j.cub.2019.02.042

Zimbres B., Furtado M.M., Jácomo A.T.A., Silveira L., Sollmann R., Tôrres N.M., Machado R.B. \& Marinho-Filho J. (2013) The impact of habitat fragmentation on the ecology of xenarthrans (Mammalia) in the Brazilian Cerrado. Landscape Ecology, 28: 259-269. https://doi.org/10.1007/s10980-012-9832-2

Zuur A.F., Ieno E.N., Walker N., Saveliev A.A. \& Smith G.M. (2009) Mixed effects models and extensions in ecology with R. Statistics for Biology and Health. New York: Springer-Verlag. 574 p. https://doi.org/10.1007/978-0-387-87458-6B 